\title{
UV-LED-Based Fluorescence and Reflectance Sensor System for Dermatological Diagnostics
}

\author{
B. Seme ${ }^{1}$, E. Wyrwich ${ }^{1}$, R. Skoczowsky ${ }^{1}$, F. Stüpmann ${ }^{2}$, M. Moschall ${ }^{2}$, G. Khazaka ${ }^{3}$, J. Schleusener ${ }^{4}$ \\ ${ }^{1}$ GMBU e.V., Felsbachstr. 7, 07745 Jena, Germany \\ 2 Silicann Systems GmbH, Schillerplatz 10, 18055 Rostock, Germany \\ ${ }^{3}$ Courage \& Khazaka Electronic $\mathrm{GmbH}$, Mathias-Brüggen-Straße 91, 50829 Köln, Germany \\ ${ }^{4}$ Charité - Klinik für Dermatologie, Venerologie und Allergologie, Charitéplatz 1, 10117 Berlin \\ seme@gmbu.de
}

\begin{abstract}
:
A fiber optical, UV-LED-based, spectroscopic fluorescence sensor system for diagnostic applications in dermatology has been developed and tested at excised human skin lesions. The system uses seven LEDs in the wavelength range $300 \ldots 430 \mathrm{~nm}$ to excite autofluorescence of different fluorophores in human skin. Additionally, the skin's diffuse reflectance is measured by means of a tungsten halogen lamp to correct reabsorptions of the fluorescence emission in the skin itself. Moreover, the system includes a dermatoscope to take photographs of the lesions. The setup is primary meant for the distinction between healthy skin and nonmelanoma skin cancer (NMSC) in vivo but may also be useful for other disorders like malignant melanomas and psoriasis. Results of first clinical ex vivo measurements are discussed.
\end{abstract}

Key words: Fluorescence, Reflectance, UV-LED, Dermatology, Skin Cancer

\section{Background motivation}

The skin is the largest organ of man. It covers our entire body and protects us against harmful factors from the environment. The skin removes waste products from the body through sweat and it also delivers vitamin $D$ with the help of UVB from the sun. But it is assumed that excessive amounts of UV irradiation as well as other environmental influences are responsible for the development of skin cancer. The most common NMSCs are basal cell carcinoma (BCC) and squamous cell carcinoma (SCC). Skin cancer incidences are increasing worldwide. At the same time, the costs of health care are increasing globally [1-3]. Thus, there is a high need for measures that prevent chronic skin diseases like cancer. Moreover, cost effective improvements of the diagnosis and the management of chronic skin diseases are necessary. A non-invasive, autofluorescence based, optical skin measurement system with UV-LED technology can contribute to this issue by reducing patient suffering due to the avoidance of unnecessary surgery and the rapid output of a diagnosis within minutes instead of days. LEDs, used as excitation light sources, are semiconductor devices that will become more and more low-cost in the future. They also enable compact and robust setups that are well manageable in the clinical practice. Compared to methods based on exogenous fluorophores, autofluorescence spectroscopy of the skin requires less treatment time, is safer and more cost-effective [4].

\section{State of the art in the field of optical NMSC diagostics}

A simple and established optical diagnostic method for melanomas and non-pigmented lesions is dermatoscopy. With the sole application of dermatoscopy only the visual appearance of the skin is assessed without taking into account its physicochemical properties. Thus, a biopsy of conspicuous skin lesions and their histopathological examination in the laboratory is customary for safe diagnostics after dermatoscopy. Two noninvasive optical systems for the diagnosis of NMSC are already on the market: Optical coherence tomography (OCT) and laser scanning microscopy (LSM). However, compared to the setup proposed here, these methods are more complex and more expensive. Other existing optical tools like "MelaFind" by Mela Sciences Inc. are only suitable for the detection of malignant melanomas (MM) and not applicable for the examination of NMSC.

Dermatological sensors for NMSC diagnostics based on autofluorescence spectroscopy have been investigated for about 20 years. In 1998 $\mathrm{H}$. Zeng et al. [8] utilized visible blue light 
(442 $\mathrm{nm}$ ) excitation to examine BCC lesions. Three years later Brancaleon et al. demonstrated that autofluorescence from tryptophan as well as collagen and elastin can be used for the diagnosis of NMSC when filtered excitation light of a xenon lamp at 295 and $350 \mathrm{~nm}$ wavelength is applied to the skin. Later, in 2014, Borisova et al. used LEDs at three excitation wavelengths $(365,385$ and $405 \mathrm{~nm}$ ) and demonstrated an excellent diagnostic accuracy and sensitivity of $>90 \%$ for the distinction between NMSC and benign lesions.

Compared to research known from the literature [4-8], the present work was carried out with seven excitation wavelengths that extend into the UVB as well as with multivariate evaluation methods. Moreover, only LED light sources were used for the fluorescence excitation. In this way, a high diagnostic sensitivity of the system is achievable at low system costs.

\section{Setup of the demonstration device}

The endogenous fluorophores of the skin are well known [4, 9-11]. Amino acids such as tryptophan can be excited in the short-wave UVC and UVB spectral range (260-300 nm). Their fluorescence emission is in the UVA. Structural proteins of the dermis such as elastin and collagen are stimulable with UVA radiation and fluoresce in the visible (blue). The enzyme and co-enzyme system NADH/NAD+ is excitable with radiation around $360 \mathrm{~nm}$ and emits around $450 \mathrm{~nm}$. Further relevant fluorophores are lipids (excitation at 340$390 \mathrm{~nm}$ and $430 \mathrm{~nm}$ ), keratin (excitation around 380-400 nm) and flavins (excitation 420$450 \mathrm{~nm}$ ). Autofluorescence spectroscopy can monitor these substances and detect conspicuous changes of the cellular matrix. In this way, additional information that may help the dermatologist to correctly evaluate a dermatoscopic finding can be provided.

The realized demonstration device specifically stimulates the endogenous fluorophores of the skin by successively applying seven suitable excitation wavelengths. Tab. 1 lists these wavelengths as well as the associated fluorophores of the skin. For each named wavelength a separate LED is used. All LEDs are located in a PC-controlled, fiber coupled module. By applying band pass filters in the LED module, excitation spectra with a width (FWHM) in the range of $10-20 \mathrm{~nm}$ are achieved. The LED module is connected to a fiber optical probe which touches the skin lesion during the measurement. The probe is held by a tripod to prevent it from exerting pressure on the sample, Fig. 2. In the measurement procedure only UVIVIS radiation doses that are harmless for the human skin and below the limit values defined in the directive 2006/25/EG of the European Union are used.

Tab. 1: Excitation wavelengths used and associated fluorophores of the skin.

\begin{tabular}{|l|l|}
\hline $\begin{array}{l}\text { Excitation- } \\
\text { wavelength } \\
\text { (peak)/nm }\end{array}$ & Skin fluorophores \\
\hline 303 & Tryptophan, Elastin \\
\hline 331 & $\begin{array}{l}\text { Elastin, Collagen, Elastin } \\
\text { cross-links }\end{array}$ \\
\hline 338 & $\begin{array}{l}\text { Elastin cross-links, Collagen, } \\
\text { Collagen cross-links, Lipids, } \\
\text { NADH }\end{array}$ \\
\hline 370 & NADH, Collagen cross-links \\
\hline 385 & NADH, Keratin, Lipids \\
\hline 403 & Keratin, Lipids \\
\hline 429 & Flavins, Lipids \\
\hline
\end{tabular}

Unfortunately the fluorescence emission of the skin is reabsorbed in the skin itself, mainly by the strong non-fluorescent [11] absorbers melanin and hemoglobin. Thus, to get an undisturbed, meaningful fluorescence spectrum from the skin, a correction is necessary. This can be done by evaluating the ratio between fluorescence and reflectance because the reflectance spectrum just indicates the spectral distribution of the absorption [12]. Therefore, besides fluorescence, the setup also measures the skin's diffuse reflectance by lighting the lesion with light of a broad-band fiber coupled halogen lamp. The reference spectrum of the reflectance is measured at a white teflon standard plate, Fig.2. For the fluorescence and reflectance detection the demonstration device includes two array spectrometers (Zeiss, MCS CCD and tec5, tecSpec CGS). Furthermore the setup comprises a dermatoscope (Courage \& Khazaka), the fiber optical probe and a PC which automatically controls the entire measuring sequence. A schematic of the system is shown in Fig. 1. Four quartz glass fibers with a diameter of $600 \mu \mathrm{m}$ are used to guide the excitation light from the LEDs and the halogen lamp to the skin and to transmit the fluorescence and the reflectance responses to the spectrometers. In order to avoid the measurement of ambient light, a sample container which can be darkened has been developed as well, Fig. 2. The entire measurement cycle of a skin lesion using seven excitation wavelengths only takes about one minute. The developed setup is primarily meant for in vivo measurements in the context of dermatoscopic examinations. Furthermore, the 
setup may also be useful for measurements during or before a skin operation to determine the exact areas to be cut out. Another potential application is the monitoring of psoriasis lesions whilst a psoriasis (photo)-therapy [13]. In this work, however, only ex vivo measurements on excised human skin lesions and its healthy surrounding are presented. Compared to in vivo measurements on humans, these measurements require a considerably lower expenditure with regard to the approvals by the local ethics committee.

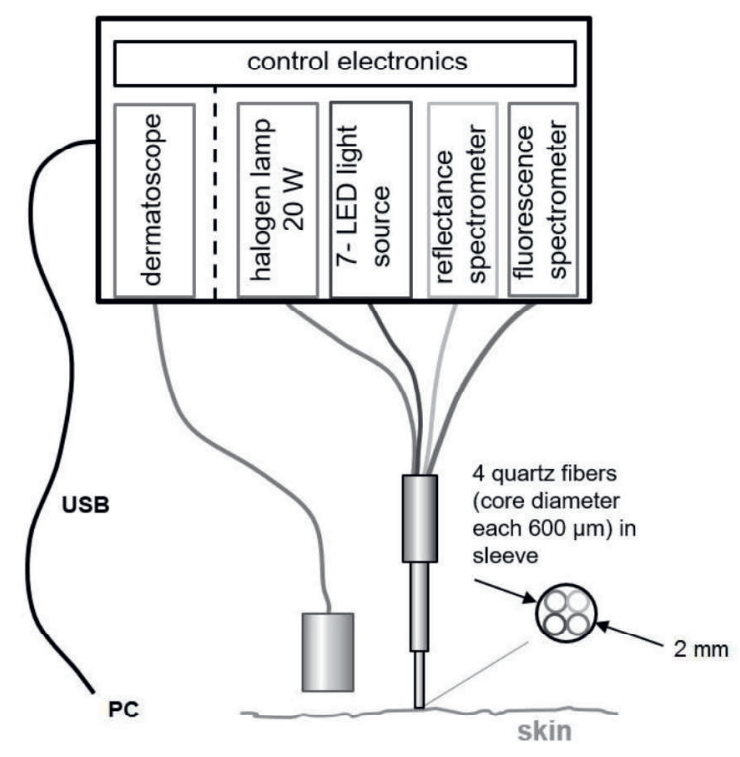

Fig. 1: Schematic of the demonstration device.

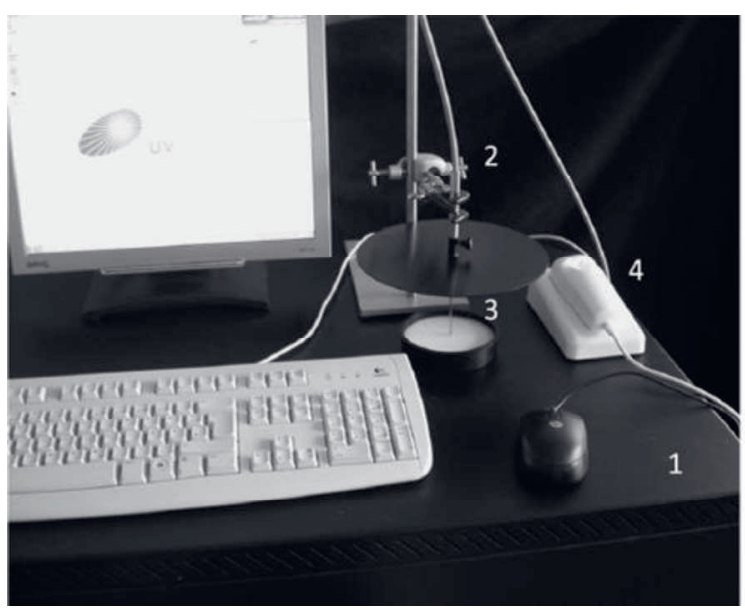

Fig. 2: Photograph of the demonstration device 1: Rack with PC, light sources and spectrometers 2: Tripod with fiber probe 3: Sample container with shutter and teflon reference in position 4: Dermatoscope

\section{First clinical measurements}

First clinical measurements with the demonstration device at eight excised BCC, four SCC, two AK (actinic keratosis) and one
MM suspicious skin lesions have been done at the Charité hospital in Berlin. The samples have been measured directly after excision and before histopathological examination of the lesion. Three of these 15 measurements had to be excluded from the further evaluation due to a very low reflectance in the 340 to $600 \mathrm{~nm}$ range $(<2.5 \%)$ which results in a non-analysable fluorescence emission. The histopathological results of all lesions are known and considered as the diagnostic gold standard, Tab.2. In each case up to six measurements on the lesion and in its surrounding have been performed and averaged to account for the skin's inhomogeneity. The standard deviation from the averaged spectra delivers a measure for the spreading of the measurements.

As an example, Fig. 3 shows measured fluorescence spectra at a SCC lesion for the seven excitation wavelengths listed in Tab.1. All discussed spectra are corrected for reabsorption in the skin (reflectance correction). To account for the different excitation powers on the skin and the different exposure times at different excitation wavelengths, counts per $\mathrm{mW}$ and per second are displayed in Fig. 3 . Moreover the spectra were corrected for the instrumental parameters of the spectrometer. In Fig. 3 fluorescence emission of four substance classes can be identified: Tryptophan emits at 330-350 nm, collagen and elastin emit mainly at 400-430 nm, NADH at 450-470 nm and the substance group keratin, lipids and flavins fluoresces at 500-540 nm. Note that emission due to collagen and elastin is not distinctive in the surrounding healthy skin whereas fluorescence of keratin, lipids and flavins is more pronounced here.

The associated reflectance spectrum of the discussed SCC lesion is presented in Fig. 4. It shows the prominent hemoglobin double peak between 500 and $600 \mathrm{~nm}$ as well as a strong hemoglobin absorption in the blue spectral range around $420 \mathrm{~nm}$. Water absorption can be seen at $980 \mathrm{~nm}$. Moreover, strong melanin absorption is superimposed, which however has no characteristic peaks in the measured wavelength range.

\section{Evaluation of the clinical measurements}

Unfortunately, due to the large number of fluorophores in the skin and due to the mentioned width of the excitation spectra applied, each of the seven LEDs excites not just one but several fluorophores, as indicated in Tab. 1. To determine the contributions of the different fluorophores, all fluorescence spectra were decomposed using the MCR-ALS algorithm [6]. The algorithm was fed with up to three characteristic input signatures as start 
values. In that way, the contributions of the mentioned classes of fluorophores to a single fluorescence spectrum and to the whole set of seven emission spectra could be calculated for each sample. These calculated contributions are also called concentrations even though no absolute concentrations e.g. in $\mathrm{mmol} / \mathrm{l}$ can be quoted. Instead we compared the concentrations of fluorophores in the lesion with that in the surrounding healthy skin by evaluating the fluorescence emission at all excitation wavelength and by calculation the ratio of the concentrations in the lesion and in the surrounding, Tab. 2. It showed that for most BCC and SCC lesions the tryptophan concentration in the tumor is increased significantly compared to surrounding skin. This is probably due to epidermal hyperproliferation [7]. At the same time, the NADH concentration is decreasing by a factor of 0.3 to 0.9 which

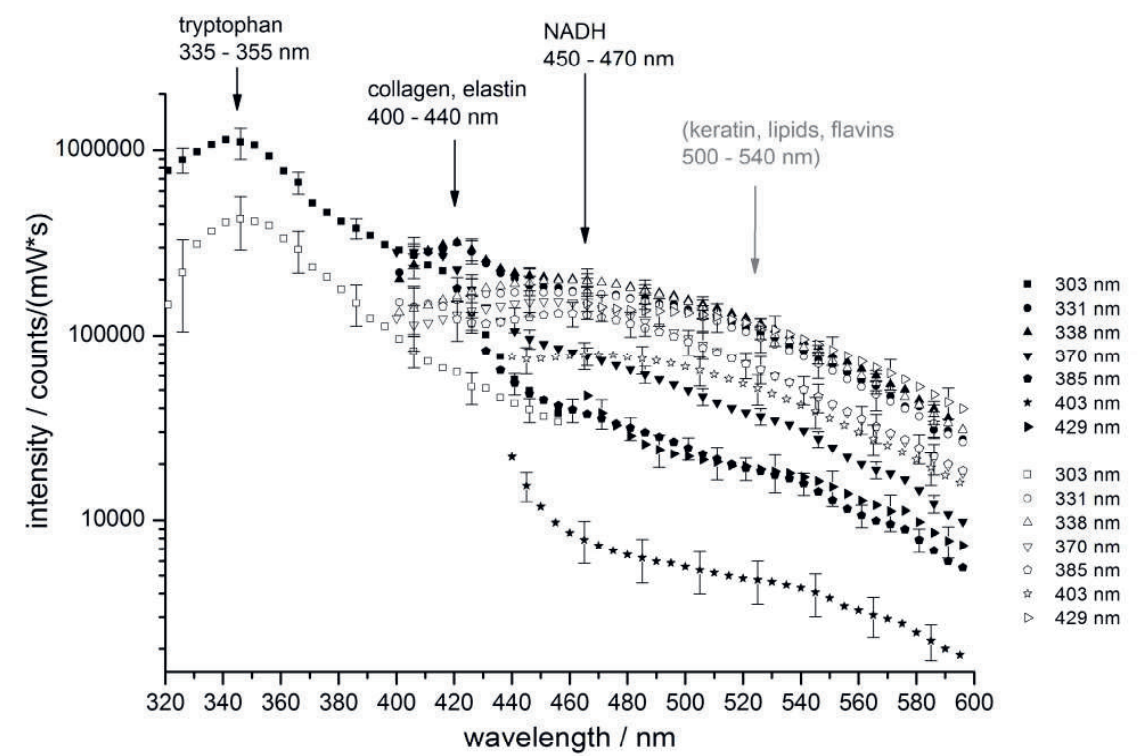

Fig. 3: Corrected fluorescence emission spectra measured at the SCC lesion P9 (black) and in its surrounding (gray). Each averaged over 5 measurements.

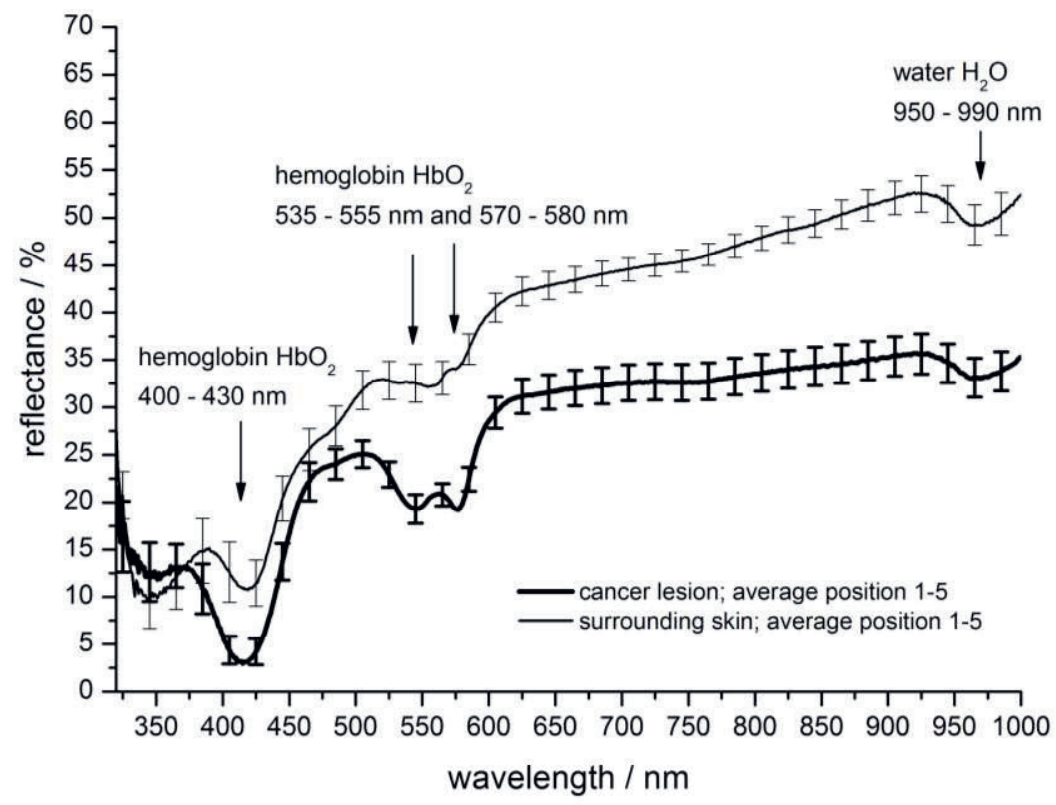

Fig. 4: Reflectance spectrum of the SCC lesion P9 and comparison with the spectrum in its surrounding. Each averaged over 5 measurements. 
indicates a change in the glycogen metabolism [5]. However, these NADH changes are less significant than the tryptophan changes, Tab. 2. Alterations of collagen, elastin, keratin, flavin and lipid concentrations are not given in the table, because they cannot be quantified. The corresponding fluorescence was not identifiable either in the lesion or in the surrounding healthy skin, Fig. 3. However, increased band depths of reflectance spectra around 545 and $575 \mathrm{~nm}$ indicate that there is an increased oxyhemoglobin concentration in most BCC and SCC lesions that may be due to tumor proliferation, Fig. 4, Tab.2.

Tab. 2: Calculated concentrations of fluorophores and band depths of reflectance spectra in lesions relative to surrounding skin. The histopathological results are given in bold text.

\begin{tabular}{|c|c|c|c|}
\hline & \multicolumn{2}{|l|}{$\begin{array}{l}\text { Fluorescence } \\
\text { spectra }\end{array}$} & \multirow{2}{*}{$\begin{array}{l}\text { Reflectance } \\
\text { spectra } \\
\mathrm{HbO}_{2} \text { band } \\
\text { depth } \\
\text { relative to } \\
\text { surrounding } \\
\text { skin }\end{array}$} \\
\hline & $\begin{array}{l}\text { tryptophan } \\
\text { concentration } \\
\text { relative to } \\
\text { surrounding } \\
\text { skin }\end{array}$ & $\begin{array}{l}\text { NADH } \\
\text { concentration } \\
\text { relative to } \\
\text { surrounding } \\
\text { skin }\end{array}$ & \\
\hline \multicolumn{4}{|c|}{ benign } \\
\hline P7 & $2.5_{-0.9}^{+3.4}$ & $0.4_{-0.1}^{+0.2}$ & $1.8_{-0.4}^{+0.5}$ \\
\hline \multicolumn{4}{|c|}{ BCC } \\
\hline P3 & $6.5_{-3.1}^{+10.3}$ & $0.6_{-0.2}^{+0.4}$ & $0.6_{-0.2}^{+0.3}$ \\
\hline P4 & $1.1_{-0.6}^{+1.1}$ & n. a. & $1.6_{-0.5}^{+0.6}$ \\
\hline P5 & $10.7_{-7.3}^{+17.0}$ & $0.8_{-0.5}^{+0.5}$ & $1.5_{-0.9}^{+1.3}$ \\
\hline P11 & $3.4_{-1.6}^{+3.1}$ & $1.4_{-0.8}^{+0.9}$ & $1.3_{-0.5}^{+0.9}$ \\
\hline P12 & $5.9_{-1.7}^{+5.7}$ & $0.7_{-0.2}^{+0.2}$ & $0.8_{-0.2}^{+0.3}$ \\
\hline P14 & $4.1_{-2.9}^{+5.9}$ & $0.3_{-0.1}^{+0.2}$ & $1.9_{-0.6}^{+1.0}$ \\
\hline P15 & $1.3_{-0.7}^{+3.2}$ & $0.9_{-0.4}^{+0.4}$ & $1.9_{-0.5}^{+0.6}$ \\
\hline \multicolumn{4}{|c|}{ SCC } \\
\hline P1 & $3.6_{-1.2}^{+5.3}$ & $0.7_{-0.4}^{+0.2}$ & $1.1_{-0.4}^{+0.5}$ \\
\hline $\mathrm{P} 2$ & $1.0_{-0.9}^{+1.2}$ & $1.2_{-0.5}^{+0.5}$ & $1.6_{-0.3}^{+0.3}$ \\
\hline P9 & $2.8_{-1.7}^{+4.8}$ & $0.6_{-0.3}^{+0.2}$ & $1.2_{-0.4}^{+0.6}$ \\
\hline \multicolumn{4}{|l|}{ MM } \\
\hline $\mathrm{P} 13$ & $5.1_{-2.9}^{+5.9}$ & $0.4_{-0.2}^{+0.1}$ & $1.1_{-0.4}^{+0.6}$ \\
\hline
\end{tabular}

Up to now only 15 measurements have been performed. But these few tests suggest that a sensitivity of the NMSC vs. benign diagnosis in the range of 70 to $90 \%$ (up to nine correct NMSC diagnoses of a total of ten NMSC Probes) should be feasible by sole application of the increasing tryptophan concentration criteria. Tab. 2 suggests that the sensitivity can be enhanced further by adding the criteria of increasing $\mathrm{HbO}_{2}$ band depth. In this case also sample P2 can be classified correctly as NMSC and a sensitivity and diagnostic accuracy $>90 \%$ could possibly be attainable. Sensitivity of dermatoscopy alone is in the same range [14], but it depends strongly on the user and its training and experience whereas the results of the fluorescence and reflectance measurements are more objective.

Our measurements at a MM lesion demonstrated that the device delivers evaluable results even in that case. The tryptophan concentration in the MM lesion was five times higher than that in the surrounding, Tab. 2.

To validate our findings and to obtain more detailed and reliable information about the reachable sensitivity of the setup much more measurements $(>100)$ and especially more readings on benign lesions (e.g. pigmented nevus) have to be performed.

\section{Conclusion}

15 excised skin lesions were analyzed with regard to their fluorescence and diffuse reflectance spectra. The reflectance spectra allowed for a reabsorption correction of the fluorescence emission. The main outcome of the fluorescence analysis is that an increase of the lesional tryptophan concentration relative to the surrounding is an important indicator for the identification of NMSC. This indicator alone should enable a sensitivity of the NMSC vs. benign diagnosis in the range of $70-90 \%$. Additional evaluations of the reflectance spectra with regard to the $\mathrm{HbO}_{2}$ band depth should allow a further increase in sensitivity up to a value $>90 \%$, but further studies are needed in this field.

We conclude that an ordinary dermatoscope can deliver more user-independent results when it is equipped with an additional system for the measurement of reflectance and tryptophan fluorescence. This additional system is inexpensive because only one UV-LED emitting around $300 \mathrm{~nm}$ and a halogen lamp are necessary for fluorescence excitation and reflectance determination. In that way, dermatoscopy can be made more dependable in a cost effective fashion.

\section{Credits}

This work has been funded by the "Bundesministerium für Forschung und Bildung (BMBF)" within the "Advanced UV for life" consortium of the "Zwanzig20" program. The clinical measurements have been done at the "Klinik für Dermatologie, Venerologie und Allergologie", Charité Universitätsmedizin Berlin, "Center of Experimental \& Applied Cutaneous Physiology" with approval from the local ethics committee. We thank Prof. J. Lademann and his team for the support. 


\section{References}

[1] Top Medical Devices and Imaging Technologies, Frost \& Sullivan, 2013

[2] Analysis of the Global Semiconductors in Medical Imaging Market, Frost \& Sullivan, 2013

[3] Global Analysis of the Semiconductors in Healthcare Market, Frost \& Sullivan, 2013

[4] E.G. Borisova, L.P. Angelova, E.P. Pavlova: Endogenous and Exogenous Fluorescence Skin Cancer Diagnostics for Clinical Applications. IEEE Journal of selected topics in quantum electronics 20, 211-222 (2014), doi: 10.1109/JSTQE.2013.2280503

[5] U. Wollina, A. Scheibe, C. Nelskamp, D. Fassler, W.-D. Schmidt: Fluorescence-remission sensoring of skin tumors: preliminary results. Skin Research and Technology 13, 463-471 (2007), doi: 10.1111/j.1600-0846.2007.00252.x

[6] J. Jaumot, R. Gargallo, A. de Juan, R. Tauler: A graphical user-friendly interface for MCR-ALS: a new tool for multivariate curve resolution in MATLAB. Chemometrics Intellig. Lab. Syst. 76, 101-110 (2005), doi: 10.1016/j.chemolab.2004.12.007

[7] L. Brancaleon et al.: In Vivo Fluorescence Spectroscopy of Nonmelanoma Skin Cancer. Photochemistry and Photobiology 73(2), 178-183 (2001), doi: 10.1562/00318655(2001)073<0178:IVFSON>2.0.CO;2

[8] H. Zeng et al.: Autofluorescence of basal cell carcinoma. Lasers in Surgery, Advanced Characterization, Therapeutics and Systems VIII, 314 (1998), doi: 10.1117/12.312300

[9] R. Na: Skin Autofluorescence in Demarcation of Basal Cell Carcinoma. PHD thesis (2001), Department of Dermatology, Copenhagen University

[10] E. Borisova, P. Pavlova, E. Pavlova, P. Troyanova, L. Avramov: Optical biopsy of human skin - a tool for cutaneous tumours' diagnosis. Int J Bioautomation 16, 53-72 (2012)

[11] N. Kollias et al.: Fluorescence Spectroscopy of Skin. Vibrational Spectroscopy 28, 17-23 (2002), doi: 10.1016/S0924-2031(01)00142-4

[12] H. Zeng et al.: Spectroscopic and Microscopic Characteristics of Human Skin Autofluorescence Emission. Photochemistry and Photobiology 61(6), 639-45 (1995), doi: 10.1111/j.17511097.1995.tb09881.x

[13] U. Wollina et al.: Targeted $307 \mathrm{~nm}$ phototherapy in psoriasis. A pilot study comparing a $307 \mathrm{~nm}$ excimer light with topical dithranol. Skin Research and Technology 18, 212-218 (2012), doi: 10.1111/j.1600-0846.2011.00556.x

[14] M. Ulrich et al.: The sensitivity and specificity of optical coherence tomography for the assisted diagnosis of nonpigmented basal cell carcinoma: an observational study, British Journal of Dermatology 173, 428-435 (2015), doi: $10.1111 /$ bjd. 13853 